\title{
Lungenbeteiligung bei primär biliärer Zirrhose
}

Karen Laudenbach, Bernd Seese

Die primär biliäre Zirrhose ist eine Autoimmunkrankheit, die gegen Mitochondrien gerichtet ist. Typische Symptome sind Juckreiz, Xanthelasmen und Rheuma-ähnliche Gelenkbeschwerden. Ungewöhnlich ist, wie im hier beschriebenen Fall, eine primär pulmonale Beteiligung: Die Patientin litt vor allem an bronchialen Symptomen bei gleichzeitig erhöhten Transaminasen.

Tab. 1 Ergebnisse einzelner Untersuchungen.

\begin{tabular}{|c|c|}
\hline Körperliche Untersuchung & $\begin{aligned} \text { - } & \text { Größe } 170 \mathrm{~cm}, 85 \mathrm{~kg}, \text { BMI } 29,4 \mathrm{~kg} / \mathrm{m}^{2} \text {, } \\
\text { - } & \text { Herzfrequenz } 85 / \mathrm{Min} \\
\text { - } & \text { Blutdruck } 92 / 68 \mathrm{mmHg} \\
\text { - } & \text { kein Fieber } \\
\text { - } & \text { Cor, Pulmo, Abdomen, Gelenke, Haut unauffällig } \\
\text { - } & \text { keine vergrößerten Lymphknoten tastbar, keine } \\
& \text { Beinödeme. }\end{aligned}$ \\
\hline $\begin{array}{l}\text { Klinisch-chemische } \\
\text { Untersuchung }\end{array}$ & 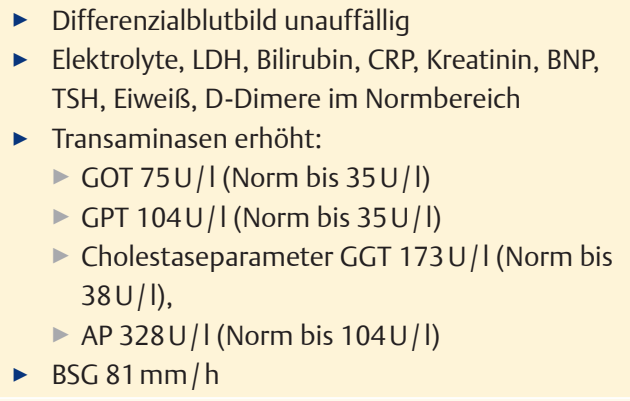 \\
\hline Blutgasanalyse & $\begin{array}{l}\text { - } \mathrm{pH} 7,4 \\
\text { } \mathrm{pCO}_{2} 35,7 \mathrm{mmHg} \\
\text { } \mathrm{PO}_{2} 74 \mathrm{mmHg} \\
\Rightarrow \text { Diffusion (DLCO) vermindert ( } 65 \% \text { Soll) }\end{array}$ \\
\hline Lungenfunktion & $\begin{array}{l}\text { Keine relevante Obstruktion, leichtgradige } \\
\text { Restriktion (TLC 70\% Soll, VC 79\% Soll) }\end{array}$ \\
\hline Immunologie & $\begin{array}{l}\text { Präzipitierende Antikörper auf Vogelfedern und } \\
\text {-kot, Penicillium notatum, Aspergillus fumigatus, } \\
\text { Alternaria alternata, Cladosporium herbarum } \\
\text { nicht erhöht } \\
\text { - IgE, Angiotensin-Converting-Emzym (ACE), } \\
\text { Interleukin-2 (IL-2), Alpha1-Antitrypsin, c-ANCA, } \\
\text { P-ANCA nicht erhöht } \\
\text { - HIV I-II negativ. }\end{array}$ \\
\hline $\begin{array}{l}\text { Bronchoalveoläre Lavage } \\
\text { (BAL) }\end{array}$ & $\begin{aligned} & \text { Lymphozyten-Anteil erhöht (55\%) } \\
& \text { - leichte Eosinophilie (9\%) } \\
& \text { T4/T8-Quotient 1,47 } \\
& \text { - Keine Viren nachweisbar (Cytomegalie, Influenza } \\
& \text { A/B, Parainfluenza 1-4, RSV, Metapneumonievi- } \\
& \text { rus, Adenovirus, Entero-, Rhino-, Parecho-, Coro- } \\
& \text { na- und Bocavirus negativ) } \\
& \text { Kein Nachweis von Pneumocystis jirovecii oder } \\
& \text { Pilzhyphen }\end{aligned}$ \\
\hline
\end{tabular}

tung hinzugekommen. In der Röntgen-ThoraxAufnahme zeigte sich eine disseminierte grobfleckige, unscharf begrenzte Zeichnungsvermehrung im Unter- und Mittelfeld der Lunge ( Abb. 1). In der hochauflösenden DünnschichtCT (HR-CT) des Thorax bestätigten sich fleckige alveoläre und interstitielle Infiltrate, teilweise konsolidierend ( $\bullet$ Abb. 2). Relevante Vorerkrankungen lagen nicht vor. Die Frau rauchte gelegentlich 1-2 Zigaretten pro Tag, nahm keine Drogen, hatte keine Haustiere und keine Allergien. Sie war Bürokauffrau.

Untersuchungen I Die körperliche Untersuchung sowie die Laborwerte waren abgesehen von erhöhten Transaminasen und Cholestaseparametern sowie erhöhter Blutsenkungsgeschwindigkeit unauffällig ( $\bullet$ Tab. 1). Die Diffusionskapazität der Lunge war mit $65 \%$ vom Sollwert vermindert und die Lungenfunktionsmessung zeigte eine leichtgradige Restriktion. Die Echokardiografie sowie die Sonografie des Abdomens blieben ohne pathologischen Befund. Ebenso war die Bronchoskopie unauffällig.

Histologie I In den transbronchialen Biopsaten zeigten sich

- eine mittelgradig chronische gering floride, gering eosinophilenhaltige Bronchitis sowie

- eine granulomatöse Entzündungsreaktion.

- Zudem bestand eine lymphozytäre Alveolitis

- und es stellte sich ein kleiner Herd intraalveolärer Fibroblasten dar.

\section{Verlauf}

Lungenresektion I Da die HR-CT, IL-2 sowie ACE untypisch für eine Sarkoidose waren, erfolgte eine minimal-invasive atypische Resektion aus Segment 9 rechts. Histologisch bestätigte sich eine mäßige Alveolitis mit nicht verkäsenden Granulomen - ein Hinweis, aber kein Beweis für eine exogen allergische Alveolitis. Granulome finden sich bei verschiedenen Erkrankungen wie z.B. Tuberkulose. Es fehlten jedoch säurefeste Stäbchen oder zentral nekrotisierende Granulome. 
Differenzialdiagnosen | Folgende mögliche Differenzialdiagnosen schlossen wir aus:

- Berylliose: keine berufliche Exposition

- Vaskulitis: histologisch nicht nachweisbar

- Histiozytosis X: CT Thorax nicht typisch

- organisierende Pneumonie: aufgrund der Alveolitis denkbar, Granulome treten dabei allerdings nicht auf

- arzneimittelinduzierte Alveolitis: anamnestischer Ausschluss

- HIV oder andere Virusinfektion: serologisch nicht nachweisbar $(\triangleright$ Tab. 1$)$

Therapie I Der T4/T8-Quotient von 1,47 bei Lymphozytose in der BAL ( $\triangleright$ Tab. 1) war weder richtungsweisend für eine Sarkoidose (Quotient $>3$ ) noch für eine exogene allergische Alveolitis (Quotient $<1,3$ ). Trotz fehlender präzipitierender Antikörper sprach die Gesamtschau der Befunde (Histologie, Lymphozyten-Anteil $>50 \%$, Bildgebung, negatives IL-2 und ACE) für eine exogen allergische Alveolitis. Die Patientin erhielt eine Behandlung mit Prednisolon:

- 2 Wochen $40 \mathrm{mg} / \mathrm{d}$,

- 2 Wochen $20 \mathrm{mg} / \mathrm{d}$,

- dann schrittweise Reduktion über 5 Monate bis zum vollständigen Absetzen.

Die Lungenveränderungen sowie die pulmonalen Symptome bildeten sich nahezu vollständig zurück. Die erhöhten Leberwerte sollten zeitnah bei einem gastroenterologischen Facharzt abgeklärt werden. Dies erfolgte jedoch nicht.

Erneute Vorstellung nach einem Jahr | Nach einem Jahr wurde die Patientin erneut stationär aufgrund von Husten, Belastungsdyspnoe und Müdigkeit aufgenommen. BSG $69 \mathrm{~mm} / \mathrm{h}$. Die HR-CT des Thorax zeigte multiple konfluierende Rundherde, DD Metastasen ( Abb. 3). Die transbronchiale Biopsie aus Ober-, Mittel- und Unterlappen rechts ergab eine mittelgradige chronische, leicht eosinophilenhaltige und gering fibrosierende Bronchitis bzw. abschnittsweise eine peribronchiale Alveolitis. BAL: Lymphozytose von 73\%; T4/T8-Quotient 1,2.

Lungenbiopsie I Die Lungenbiopsie erbrachte auch dieses Mal keine eindeutige Klärung. Die multiplen konfluierenden Rundherde in der Bildgebung waren jetzt nicht mehr typisch für die zuvor vermutete exogen allergische Alveolitis. Aufgrund des Alters der Patientin, der Konzentration des löslichen IL-2-Rezeptors (1481U/l; Norm $<623 \mathrm{U} / 1$ ), der weiterhin erhöhten Transaminasen (GOT 84U/l, GPT 132U/1) und Cholestaseparameter (GGT 99U/1, AP 428U/1) sowie der zuvor nachgewiesenen Granulome dachten wir an eine nodöse Form der Sarkoidose mit Leberbeteiligung.

Abklärung der erhöhten Leberwerte I In der gastroenterologischen Fachabteilung wurden nun die erhöhten Leberwerte abgeklärt. Weil die klinischen Beschwerden nicht richtungsweisend für

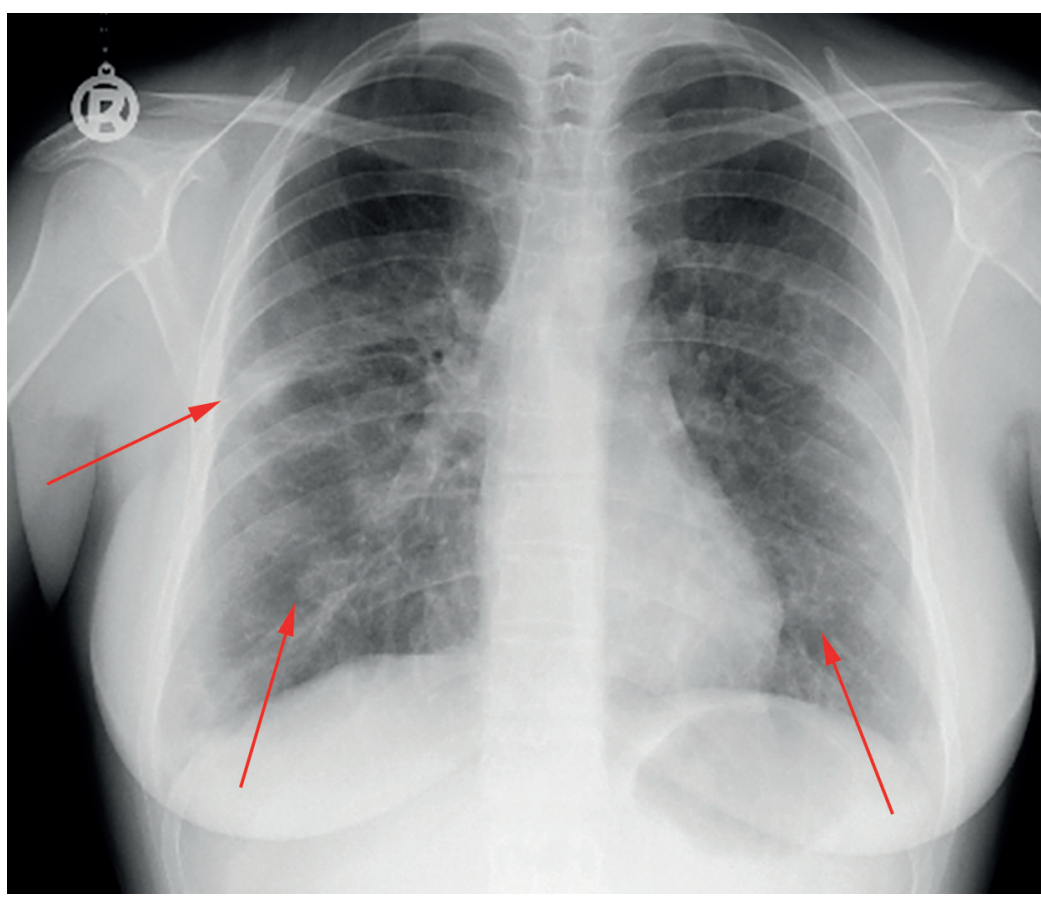

Abb. 1 Röntgen-Thorax bei Aufnahme der Patientin zeigt

eine spezifische Lebererkrankung waren, entschied man sich zur laparoskopischen Histologiegewinnung bei Verdacht auf eine sarkoidoseähnliche Erkrankung. Bereits makroskopisch ergab sich das typische Bild einer frühen Form der primär biliären Zirrhose. Diese Diagnose wurde bestätigt durch

- die Histologie (PBC Grad I),

- den hochtitrigen Nachweis antimitochondrialer Antikörper (AMA) Subtyp Anti M2 (>1:640), - erhöhte IgM-Werte( $9,8 \mathrm{~g} / \mathrm{dl}$, Norm bis 2,3 g/ dl). In einem Referenzlabor wurde ein Overlap-Syndrom mit einer Autoimmunhepatitis ausgeschlossen. Unter Behandlung der PBC mit Ursodeoxycholsäure waren die Leberwerte nach 2 Monaten rückläufig (GOT 35U/1, GPT 57U/1, AP 152U/l, Computertomografie zeigt fleckige und alveoläre Infiltrate (rote Pfeile), teilweise konsolidierend (schwarzer Pfeil).

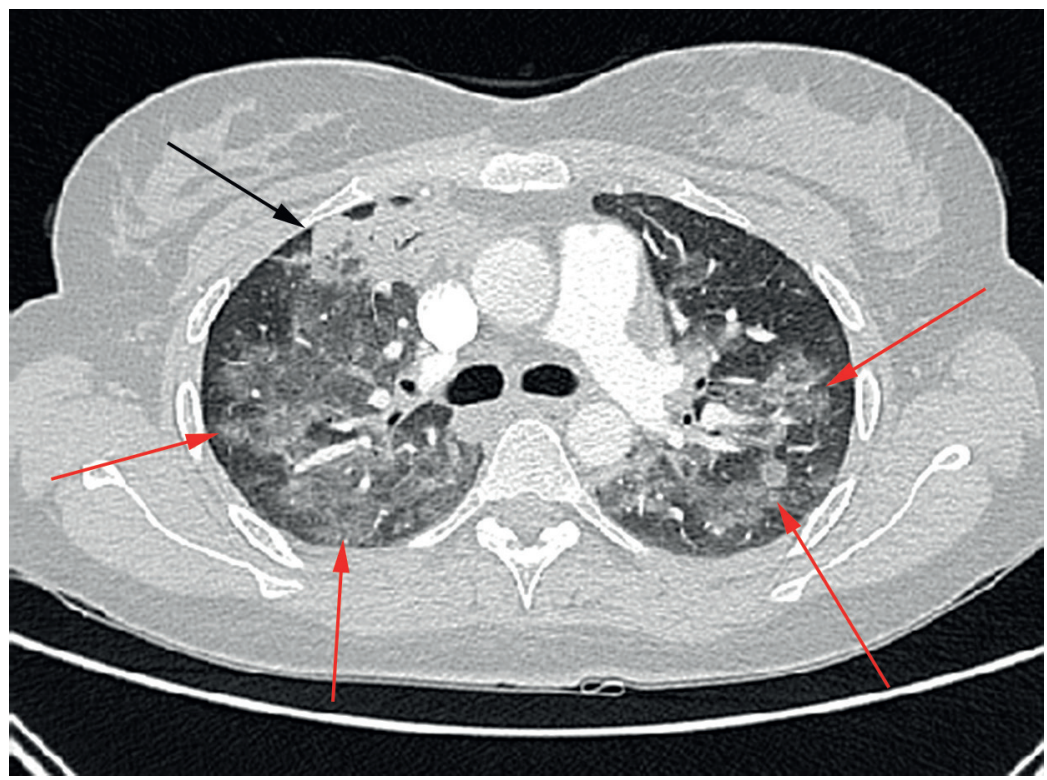




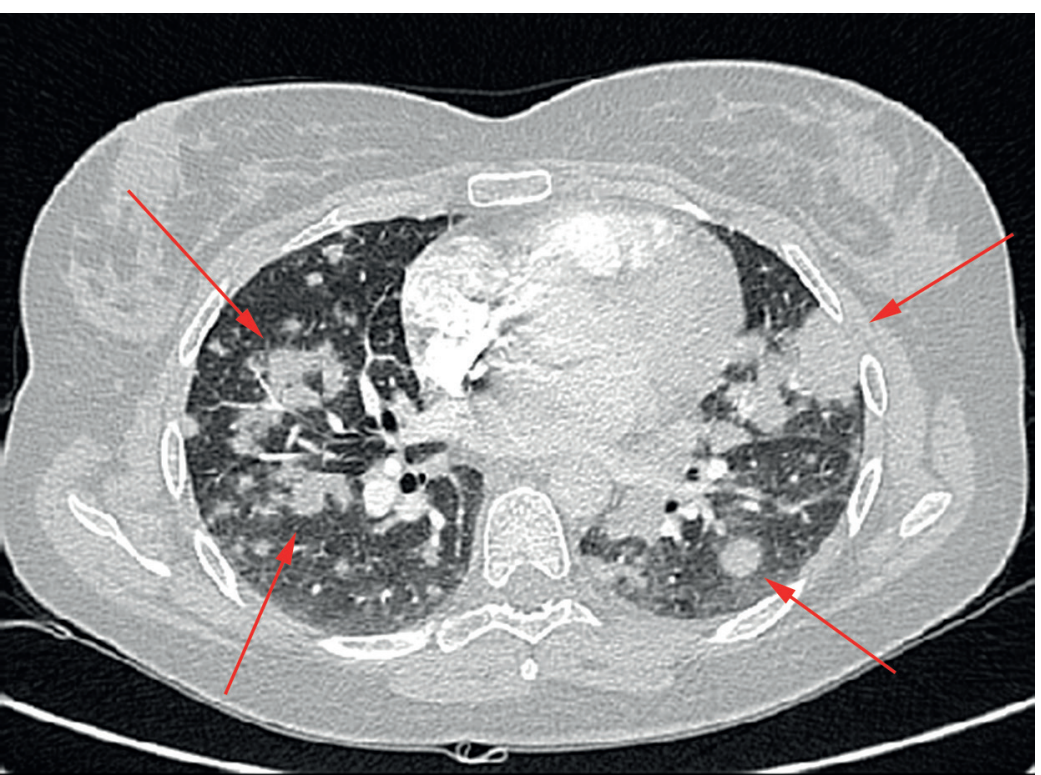

Abb. 3 HR-CT des Thorax. Es sind multiple konfluierende Rundherde sichtbar (Pfeile).

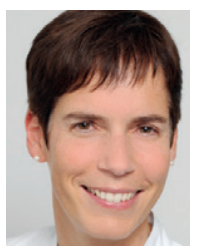

GGT 43U/1). Die Lungenherde waren 3 Monate nach Behandlungsbeginn ohne zusätzliches systemisches Kortison fast vollständig regredient. Die Patientin war pulmonal beschwerdefrei.

\section{Diskussion}

PBC und Lungenerkrankungen | Die Koinzidenz der primär biliären Zirrhose (PBC) mit verschiedenen Lungenerkrankungen ist bekannt. Bei einer PBC wurden z. B bereits beschrieben:

- interstitielle Lungenerkrankungen bzw. Sarkoidose-ähnliche Granulome [1-6]

- Bronchiolitis-organisierende Pneumonie und lymphozytäre interstitielle Pneumonitis [7]

- nekrotisierende sarkoidale Granulomatose [8]

Diffusionskapazität häufig betroffen | Einige Studien deuten darauf hin, dass bei einer Lungenbeteiligung der PBC die Diffusionskapazität der Lunge häufiger als die Lungenfunktion betroffen ist. Dies war auch bei unserer Patientin der Fall. Nach Behandlung der PBC verbesserte sich die Diffusion von $65 \%$ auf $77 \%$ Soll.

Dr. Bernd Seese ist Ärztlicher Direktor und Chefarzt der Pneumologie am Thoraxzentrum Bezirk Unterfranken, Münnerstadt b.seese@tzbu.de

Interessenkonflikt Die Autoren geben an, dass kein Interessenkonflikt besteht

DOI 10.1055/s-0041-100353 Dtsch Med Wochenschr 2015; 140: 264-266

(c) Georg Thieme Verlag KG . Stuttgart · New York · ISSN 0012-0472

- Bei 24 von 61 Patienten mit unterschiedlichen Stadien einer PBC war, obwohl die Patienten keine pulmonalen Beschwerden hatten, die Diffusionskapazität ohne Korrelation zum Raucherstatus reduziert [9].

- Von 178 Patienten mit PBC wiesen 15,7\% eine interstitielle Lungenerkrankung auf [5]. 53,6\% von ihnen litten wie unsere Patientin an Atemnot und Husten. Bei 88,2\% dieser symptomatischen Patienten wurden ebenfalls eine Diffusionsstörung und eine restriktive Lungenfunktion festgestellt. Symptomatische Patienten waren im Schnitt älter und die BSG war höher als bei den Patienten ohne interstitielle Lungenerkrankung. Bei unserer Patientin war die Totalkapazität mit 70\% Soll leicht erniedrigt, die hohe BSG sowie erhöhte IgG-Globuline waren zudem Ausdruck hoher Entzündungsaktivität.

- Jastrzebski et al. [10] analysierten die BAL bei 13 Patienten mit PBC. Eine Lymphozytose (>15\%) fand sich bei $38 \%$. Das Kollektiv wies eine unauffällige Lungenfunktion auf, 38\% hatten eine Diffusionsstörung.

Weitere häufige Begleiterkrankungen | Ein Raynaud-Phänomen ist ein Risikofaktor für die Entwicklung einer interstitiellen Lungenerkrankung bei PBC [5]. Ferner beobachteten Liu et al. [3], dass ein Sjögren-Syndrom gehäuft im Zusammenhang mit einer interstitiellen Lungenerkrankung auftritt. Beide Erkrankungen lagen bei unserer Patientin nicht vor.

Therapieansprechen | Unsere Patientin sprach relativ rasch auf die immunsuppressive Kortisonbzw. auf die Ursodeoxycholsäure-Therapie an die pulmonalen Symptome und die Lungenherde gingen zurück. Die Literatur bestätigt dies.

- Frechen et al. [8] berichteten von einer deutlichen Regredienz der Rundherde bereits nach 2 Wochen Kortison-Behandlung mit $40 \mathrm{mg} / \mathrm{d}$, dann Reduktion auf $30 \mathrm{mg} / \mathrm{d}$.

- Auch andere Autoren bestätigten diese relativ rasche pulmonale Besserung unter Kortison [1, 7].

Diagnosestellung erschwert | Die Gesamtschau der Befunde unserer Patientin spricht für eine pulmonale Beteiligung bei PBC. Die Diagnosestellung wurde dadurch erschwert, dass klassische Symptome der PBC wie Juckreiz, Xanthelasmen, Hepatosplenomegalie, Hyperpigmentation [11] fehlten und die erhöhten Leberwerte zwar zur Kenntnis genommen, aber nicht zeitgerecht abgeklärt wurden. Letztlich lässt sich die Lungenbeteiligung bei PBC zwar nicht beweisen, aber die Tatsache, dass sich die ausgeprägte pulmonale Symptomatik und die multiplen Rundherde in der Bildgebung allein durch Behandlung der PBC fast vollständig zurückbildeten, sprechen dafür.

\section{Konsequenz für Klinik und Praxis}

- Bei einer primär biliären Zirrhose regelmäßig Lungenfunktion und Diffusion prüfen, um eine pulmonale Beteiligung frühzeitig zu erkennen. generell umfangreich und zeitnah abklären, insbesondere wenn auch unklare interstitielle Lungenveränderungen oder eine sarkoidale Granulomatose vorliegen.

\section{Literatur}

1 Bartosiewicz M, Siemion-Szcześniak I, Jedrych M et al. Interstitial lung disease in patients with primary biliary cirrhosis. Pneumonol Alergol Pol 2012; 80: 471-481.

2 Kayser K, Bohrer M, Kayser C et al. Alteration of human lung parenchyma associated with primary biliary cirrhosis. Zentralbl Pathol 1993; 139: 377-380

Vollständiges Literaturverzeichnis unter

http://dx.doi.org/10.1055/s-0041-100353 
3 Liu B, Zhang FC, Zhang ZL, Zhang W, Gao LX. Interstitial lung disease and Sjögren's syndrome in primary biliary cirrhosis: a causal or casual association? Clin Rheumatol 2008; 27: 1299-1306.

4 Płodziszewska M, Słodkowska J, Orłowski T, Pawlicka L, Rowińska-Zakrzewska E. Primary biliary cirrhosis with sarcoid-like infiltrations in the lung. Pneumonol Alergol Pol 1998; 66: 330-336.

5 Shen M, Zhang F, Zhang X. Primary biliary cirrhosis complicated with interstitial lung disease: a prospective study in 178 patients. | Clin Gastroenterol 2009; 43: 676-679

6 Wallace JG Jr, Tong MJ, Ueki BH, Quismorio FP. Pulmonary involvement in primary biliary cirrhosis. J Clin Gastroenterol 1987; 9: 431-435

7 Strobel ES, Bonnet RB, Werner P, Schaefer HE, Peter HH Bronchiolitis obliterans organising pneumonia and primary biliary cirrhosis-like lung involvement in a patient with primary biliary cirrhosis. Clin Rheumatol 1998; 17: 246-249.
8 Frechen D, Cornelissen C, Schreiner K, Jäkel J, Krüger S. Nekrotisierende sarkoidale Granulomatose der Lunge bei einer Patientin mit primär biliärer Leberzirrhose. Dtsch Med Wochenschr 2010; 135: 1733-1736

9 Costa C, Sambataro A, Baldi S et al. Primary biliary cirrhosis: lung involvement. Liver 1995; 15: 196-201

10 Jastrzebski DT, Musialik JA, Ziora DI et al. Lung function tests and bronchoalveolar lavage (BAL) findings in patients with primary biliary cirrhosis. Wiad Lek 2002; 55: 516-524.

11 Uddenfeldt P, Bjerle P, Danielsson A, Nyström L, Stjernberg $\mathrm{N}$. Lung function abnormalities in patients with primary biliary cirrhosis. Acta Med Scand 1988; 223: 549-555 